\title{
PlantPhos: using maximal dependence decomposition to identify plant phosphorylation sites with substrate site specificity
}

\author{
Tzong-Yi Lee*, Neil Arvin Bretaña and Cheng-Tsung Lu
}

\begin{abstract}
Background: Protein phosphorylation catalyzed by kinases plays crucial regulatory roles in intracellular signal transduction. Due to the difficulty in performing high-throughput mass spectrometry-based experiment, there is a desire to predict phosphorylation sites using computational methods. However, previous studies regarding in silico prediction of plant phosphorylation sites lack the consideration of kinase-specific phosphorylation data. Thus, we are motivated to propose a new method that investigates different substrate specificities in plant phosphorylation sites.

Results: Experimentally verified phosphorylation data were extracted from TAIR9-a protein database containing 3006 phosphorylation data from the plant species Arabidopsis thaliana. In an attempt to investigate the various substrate motifs in plant phosphorylation, maximal dependence decomposition (MDD) is employed to cluster a large set of phosphorylation data into subgroups containing significantly conserved motifs. Profile hidden Markov model (HMM) is then applied to learn a predictive model for each subgroup. Cross-validation evaluation on the MDD-clustered HMMs yields an average accuracy of $82.4 \%$ for serine, $78.6 \%$ for threonine, and $89.0 \%$ for tyrosine models. Moreover, independent test results using Arabidopsis thaliana phosphorylation data from UniProtKB/SwissProt show that the proposed models are able to correctly predict $81.4 \%$ phosphoserine, $77.1 \%$ phosphothreonine, and $83.7 \%$ phosphotyrosine sites. Interestingly, several MDD-clustered subgroups are observed to have similar amino acid conservation with the substrate motifs of well-known kinases from Phospho.ELM-a database containing kinase-specific phosphorylation data from multiple organisms.

Conclusions: This work presents a novel method for identifying plant phosphorylation sites with various substrate motifs. Based on cross-validation and independent testing, results show that the MDD-clustered models outperform models trained without using MDD. The proposed method has been implemented as a web-based plant phosphorylation prediction tool, PlantPhos http://csb.cse.yzu.edu.tw/PlantPhos/. Additionally, two case studies have been demonstrated to further evaluate the effectiveness of PlantPhos.
\end{abstract}

\section{Background}

Protein phosphorylation is the most widespread and well-studied post-translational modification in eukaryotic cells. It is one of the most prevalent intracellular protein modifications that influence numerous cellular processes [1]. It has been estimated that one-third to one-half of all proteins in a eukaryotic cell are phosphorylated [2]. Furthermore, protein phosphorylation, catalyzed by specific kinases, plays crucial regulatory

\footnotetext{
* Correspondence: francis@saturn.yzu.edu.tw

Department of Computer Science and Engineering, Yuan Ze University, Chungli 320, Taiwan
}

roles in intracellular signal transduction. Networks composed of proteins and small molecules that transmit information from the cell surface to the nucleus are ultimately affected by transcriptional changes [3]. An estimated $1 \%$ to $3 \%$ of functional eukaryotic genes encode protein kinases; this suggests that they are involved in many aspects of cellular regulation and metabolism [4]. However, a full understanding on the mechanism of intracellular signal transduction remains a major challenge in cellular biology.

Protein phosphorylation regulates various cellular processes not only in mammals but also in plants. It is

\section{Biomed Central}


reported that the regulation of carbon and nitrogen metabolism in plants is driven by phosphorylation [5]. Phosphorylation is also involved in modulating a sucrose phosphate synthase enzyme which controls the signaling pathway for the process of sucrose synthesis in plants [6]. Phosphorylation also aids in modulating the plant process of synthesizing Ammonia, an organic compound which is required to give energy to certain organs which are not able to photosynthesize [6]. Furthermore, protein phosphorylation is involved in the process of plant growth and plant response to stress [6,7]. Stone et al. have identified a number of plant kinases; however, the precise functional roles of specific protein kinases were not widely elucidated [4].

Due to the interest of the scientific community in further understanding the process of phosphorylation, mass spectrometry-based proteomics have been used to enable the large-scale mapping of in vivo phosphorylation sites [8]. With this, several databases have been proposed to store experimentally verified phosphorylation sites with catalytic kinases, such as Phospho.ELM [9], PhosphoSite [10], UniProtKB/Swiss-Prot [11], PHOSIDA [12], and dbPTM [13,14]. While most resources focus on phosphorylation sites in mammalian organisms, there are some databases which store phosphorylation sites in plants such as PhosPhAt [15], P3DB [16] and TAIR [17]. PhosPhAt consolidates knowledge of mass spectrometry-based identified phosphorylation sites in Arabidopsis thaliana and offers a phosphorylation site prediction tool specifically trained on experimentally identified Arabidopsis thaliana phosphorylation motifs [15]. P3DB provides a resource of protein phosphorylation data from multiple plants. Moreover, a phosphopeptide BLAST browser was implemented to allow users to query the database for phosphopeptides similar to protein sequences of their interest [16]. TAIR maintains a database of genetic and molecular data for Arabidopsis thaliana [17]. Protein data stored in TAIR includes the complete protein sequence along with phosphorylation site annotations.

Due to the high complexity and difficulty of phosphorylation site identification using mass spectrometry, a number of mammalian protein phosphorylation prediction tools have been developed using different methods and yielding various predictive performance. KinasePhos 1.0 [18,19], incorporated profile HMM for identifying kinase-specific phosphorylation site prediction, whose overall predictive accuracy is about $87 \%$. Version 2.0 of KinasePhos [20] incorporated support vector machine (SVM) with the protein coupling pattern to identify phosphorylation sites for 58 kinase groups. NetPhosK [21] applied an artificial neural network algorithm to predict $17 \mathrm{PK}$ groups-specific phosphorylation sites. Scansite 2.0 [22] identified short protein sequence motifs that are recognized by modular signaling domains, phosphorylated by serine/threonine, tyrosine kinases or those that mediate specific interactions with protein or phospholipid ligands. GPS $[23,24]$ is a groupbased phosphorylation site prediction and scoring platform which clusters 216 unique protein kinases in 71 groups. PPSP [25] developed an approach based on Bayesian decision theory for predicting the potential phosphorylation sites accurately for around 70 protein kinase groups. PHOSIDA [12], incorporated SVM with surface accessibility and evolutionary conservation, made $91.75 \%, 81.06 \%$, and $76.19 \%$ accuracies in serine, threonine, and tyrosine, respectively.

With regard to plant phosphorylation prediction, PhosPhAt [15] has utilized a set of 802 experimentally validated phosphoserine sites to develop a classifier of SVM for identifying pSer sites in Arabidopsis thaliana. This yielded an area under curve rate of around 0.81 on a redundant TAIR7 [17] protein dataset. More recently, Gao et al. [26] incorporated protein sequence information and protein disordered regions, and integrated $\mathrm{k}$ nearest neighbor and SVM for predicting phosphorylation sites. Their method utilized the PhosPhAt dataset of pSer in Arabidopsis thaliana and the TAIR7 nonredundant protein database. However, these works do not predict phosphorylation sites according to plant substrate site specificity [26]. Therefore, there is a need to investigate the various substrate site specificities in plants and utilize this information for predicting kinasespecific plant protein phosphorylation sites.

Information regarding protein kinases that phosphorylate substrates in plants is very limited. Based on the collection of experimentally verified plant phosphorylation sites from TAIR9 and UniProtKB/Swiss-Prot, phosphorylation sites are not annotated with its corresponding kinase. Due to this limitation, majority of the published methods for computationally identifying kinase-specific phosphorylation sites are trained mainly by using data from non-plant organisms. This study aims to analyze plant phosphorylation sites, investigate substrate site specificity in plants, and most importantly, present a novel method for identifying potential phosphorylation sites in plant proteins using the available substrate site specificity information. This work applies maximal dependence decomposition (MDD) [27] to cluster all phosphorylated fragments into subgroups presenting meaningful and statistically significant site specificity. MDD was firstly used to group the splice sites during the identification process of splice site prediction [28]. A large group of aligned sequences can be moderated into subgroups that capture the most significant dependencies between positions. Huang et al. [19] have applied MDD to improve the prediction performance of PKA, PKC, and CK2 kinase groups. In this study, MDD 
is adopted to investigate various substrate specificities of plant phosphorylation sites. Additionally, the motif of each MDD-clustered subgroup is compared to the substrate motifs of known kinases in Phospho.ELM [9]-a database for integrating comprehensive information of kinase-specific phosphorylation sites from multiple organisms. According to a five-fold cross-validation evaluation, models trained with MDD-clustered subgroups could improve predictive accuracy as compared to models trained without the application of MDD clustering. Furthermore, an independent data set is used to further evaluate the effectiveness of the models that achieve the best accuracy during cross-validation. Finally, the MDDclustered models are adopted to implement an effective web-based tool, namely PlantPhos, for identifying plant phosphorylation sites with substrate motifs that may potentially be recognized by plant kinases. The prediction tool and the data used in this study can be available at http://csb.cse.yzu.edu.tw/PlantPhos/.

\section{Materials and methods}

Figure 1 depicts the system flow of this study. The method consists of the following processes: data collection, redundancy removal, data clustering by MDD, model learning and cross-validation, and independent testing. The details of each process are described as follows.

\section{Data collection and preprocessing}

With reference to PhosPhAt [15], this work obtains plant protein phosphorylation data from the TAIR9 database [17]. TAIR9 consists of 3006 phosphorylation data from the plant species Arabidopsis thaliana. The database contains annotations of the phosphorylated sites in each plant protein data. Based on the concept of binary classification, the residues serine $(\mathrm{S})$, threonine $(\mathrm{T})$, and tyrosine $(\mathrm{Y})$ which are annotated as phosphorylation sites in the database are regarded as positive data. On the other hand, with reference to previous works $[18,26], \mathrm{S}, \mathrm{T}$, and $\mathrm{Y}$ residues which are not annotated as phosphorylated are extracted as negative data. In order to investigate the surrounding residues of phosphorylation sites in a comprehensive manner, sequence fragments are extracted using a window size of 21 with $S$, $\mathrm{T}$, or $\mathrm{Y}$ as the central residue. As presented in Table 1, this resulted in 2516 positive data and 97965 negative data for S; 382 positive data and 51434 negative data for $\mathrm{T}$; and 108 positive data and 26405 negative data for $\mathrm{Y}$.

Redundant sequence fragments or sequences which contain the same amino acids on each corresponding position in both the positive data sets and negative data sets are removed. In the case of redundant sequence fragments found in both positive and negative data sets, the sequence in the negative data set is removed and the sequence in the positive data set is retained as shown in the redundancy removal step of Figure 1. The removal of redundant data resulted in 2506 positive fragments and 87877 negative fragments for S; 378 positive fragments and 10402 negative fragments for $\mathrm{T}$; and 108 positive fragments and 1681 negative fragments for $Y$. Since the number of the negative fragments is much greater than the number of the corresponding positive fragments, the number of data is balanced by obtaining the same number of negative fragments based on the number of positive fragments. The idea of balancing the negative data with the positive data is done in reference to PhosPhAt [15] which uses random selection to obtain a balanced data set. However, in order to avoid an unstable prediction performance through a non-representative data set derived using random selection, the $K$ means clustering method [29] is used for this study. $K$ means clustering selects well-represented data from a large data set in order to achieve a more globalized sample. A data point which has a minimal distance from other data points surrounding it is selected as a representative data. For this study, $K$-means clustering is performed based on sequence identity. The value of $K$ which denotes the number of samples to be obtained from the negative data set is defined by the number of the corresponding positive data. This resulted in a data set consisting of 2506 positive and negative $\mathrm{S}$ fragments; 378 positive and negative $\mathrm{T}$ fragments; and 108 positive and negative $Y$ fragments. Table 1 shows the final number of positive and negative fragments in $\mathrm{S}, \mathrm{T}$, and $\mathrm{Y}$ used for this study.

\section{Data clustering by maximal dependence decomposition}

One of the aims of this study is to investigate the substrate site specificity of plant phosphorylation sites based on amino acid sequences. In order to explore the conserved motifs from a large data set, MDD is applied to cluster all phosphorylated fragments into subgroups, which can show statistically significant site specificity. MDD [28] is a methodology to group a set of aligned signal sequences to moderate a large group into subgroups that capture the most significant dependencies between positions. In previous studies [28], MDD was proposed to group splice sites during the identification process of splice site prediction. However, in this work, we group protein sequences instead of nucleotides. MDD adopts chi-square test $\chi^{2}\left(\mathrm{~A}_{\mathrm{i}}, \mathrm{A}_{\mathrm{j}}\right)$ to evaluate the dependence of amino acid occurrence between two positions, $A_{i}$ and $A_{j}$, which surround the phosphorylation site. In order to extract motifs that have conserved biochemical property of amino acids when doing MDD, we categorize the twenty types of amino acids into five groups: neutral, acid, basic, aromatic, and imino groups, as shown in Additional file 1, Table S1. Then, a 


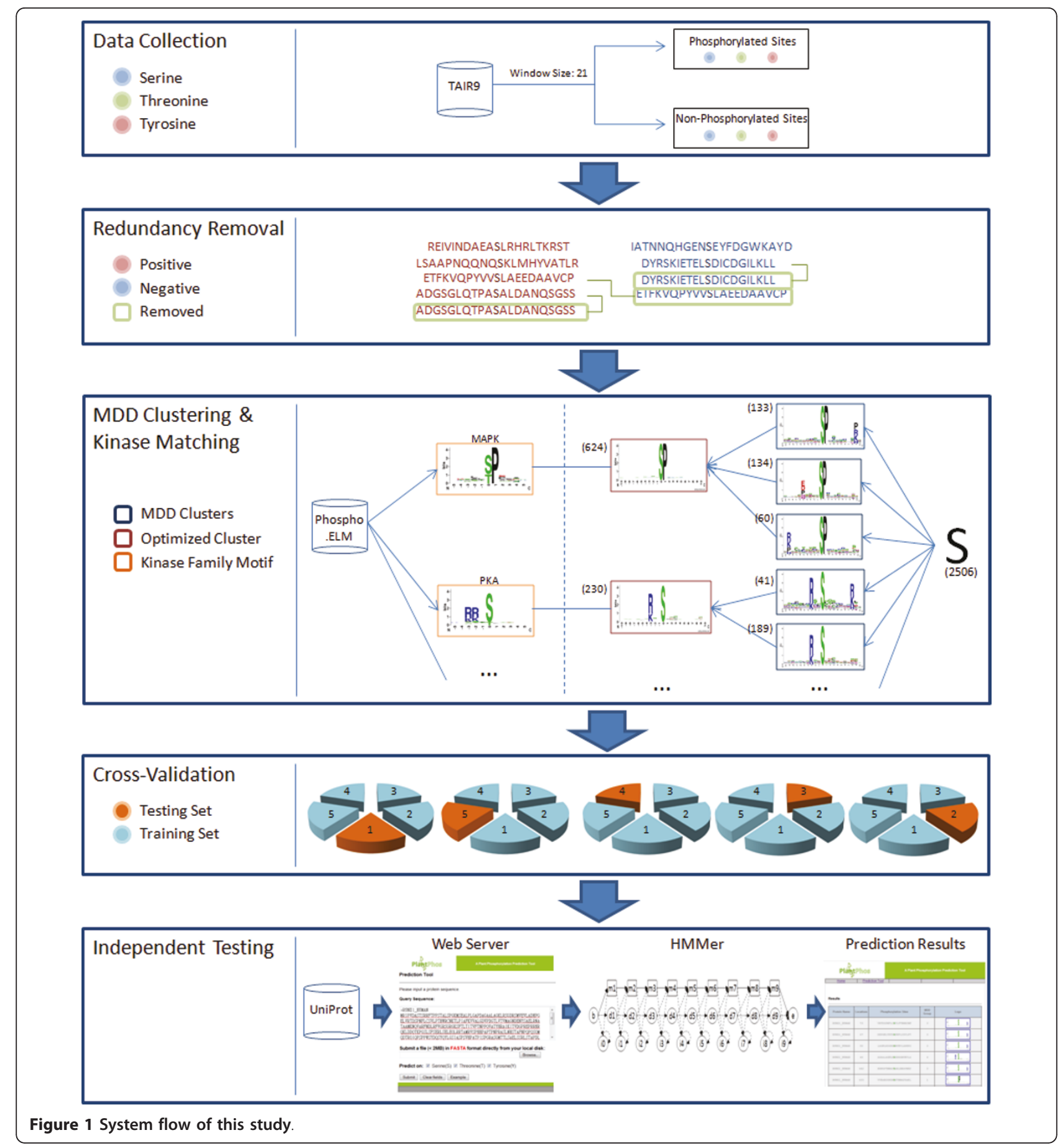

contingency table of the amino acids occurrence between two positions is constructed, as presented in Additional file 1, Figure S1. The chi-square test is defined as:

$$
\chi^{2}\left(\mathrm{~A}_{\mathrm{i}}, \mathrm{A}_{\mathrm{j}}\right)=\sum_{m=1}^{5} \sum_{n=1}^{5} \frac{\left(X_{m n}-E_{m n}\right)^{2}}{E_{m n}}
$$

where $X_{m n}$ represents the number of sequences that have the amino acids of group $m$ in position $A_{i}$ and have the amino acids of group $n$ in position $A_{j}$, for each pair $\left(A_{i}, A_{j}\right)$ with $i \neq j . E_{m n}$ is calculated as $\frac{X_{m R} \cdot X_{C n}}{X}$, where $X_{m R}=X_{m 1}+\ldots+X_{m 5}, X_{C n}=X_{1 n}+\ldots+X_{5 n}$, and $X$ denotes the total number of sequences. If a strong dependence is detected (defined as a $X^{2}$ value is larger 
Table 1 Data statistics of the experimentally verified phosphorylation sites collected from TAIR9 protein database

\begin{tabular}{ccccc}
\hline Phosphorylated residue & Type of Data & Original data & Non-redundant data & Balanced data \\
\hline S & positive & 2516 & 2506 & 2506 \\
\cline { 2 - 5 } & negative & 97965 & 87877 & 378 \\
\cline { 2 - 5 } & positive & 382 & 10402 & 378 \\
\hline T & negative & 51434 & 108 & 108 \\
\cline { 2 - 5 } & positive & 108 & 1681 & 108 \\
\end{tabular}

than 34.3, corresponding to a cutoff level of $P=0.005$ with 16 degrees of freedom) between two positions, then the process is continued as described by Burge and Karlin [28]. As illustrated in Additional file 1, Figure S1, it can be observed that position +1 has the maximal dependence with the occurrence of imino amino acids. Subsequently, all data can be divided into two subgroups where one has the occurrence of imino amino acids in position +1 while the other does not have the occurrence of imino amino acids in position +1 . MDD clustering is a recursive process which divides the positive sets into tree-like subgroups. When applying MDD to cluster the sequences in the positive set, a parameter, i. e., the minimum-cluster-size, should be set. If the size of a subgroup is less than the minimum-cluster-size, the subgroup will not be divided any further. The MDD process terminates until all the subgroup sizes are less than the value of the minimum-cluster-size. With reference to previous works that utilize MDD [18], there exists no set values for the parameters of MDD clustering. In order to obtain an optimal minimum cluster size, MDD clustering is executed using various values. Each subgroup is represented using WebLogo [30] to graphically visualize the corresponding substrate motif. The resulting clusters are then analyzed as to whether or not they contain significant conserved motifs. Subgroups with very similar motifs are further grouped together into a single cluster in order to provide more meaningful groups and avoid redundant clusters as shown in the MDD clustering step of Figure 1.

\section{Model learning and evaluation}

In this work, profile HMM is learned from the site sequences of each optimized MDD-clustered subgroups. An HMM describes a probability distribution over a potentially infinite number of sequences [31]. It can also be used to detect distant relationships between amino acids sequences. Here, we use the software package HMMER version 2.3.2 [31] to build the profile HMMs, to calibrate the HMMs, and to search the putative phosphorylation sites against the protein sequences. HMM builds a model based on positive instances of a class; thus, in this study, only positive data were utilized to build a model. The MDD-clustered sets of the phosphorylation sites are taken as training sets to learn the HMMs. One HMM is built for each MDD-clustered subgroup.

For each model of the MDD-clustered subgroups, a threshold parameter is selected as a cut-off value in identifying potential positive data from a query [31]. An optimized threshold is selected as the value which gives the most optimal cross-validation performance for each training model. To search the hits of a HMM, HMMER returns both a bit score and an expectation value (Evalue). The bit score is the base two logarithm of the ratio between the probability that the query sequence is a significant match and the probability that it is generated by a random model. The E-value represents the expected number of sequences with a score greater than or equal to the returned HMMER bit scores. A search result with an HMMER bit score greater than the threshold parameter is taken as a positive prediction. While decreasing the bit score threshold favors finding true positives, increasing the bit score threshold favors finding true negatives.

Cross-validation is an important evaluation procedure prior to the application of a predictor [32]. The predictive performance of the constructed models is evaluated by performing five-fold cross validation. The training data is divided into five groups by splitting each dataset into five approximately equal sized subgroups. During cross-validation, each one of the five subgroups is regarded as the validation set in turn, and the remainder is regarded as the training set. Next, the following measures of predictive performance of the trained models are calculated: Precision $($ Pre $)=\mathrm{TP} /(\mathrm{TP}+\mathrm{FP})$, Sensitivity $(\mathrm{Sn})=\mathrm{TP} /(\mathrm{TP}+\mathrm{FN})$, Specificity $(\mathrm{Sp})=\mathrm{TN} /(\mathrm{TN}+\mathrm{FP})$, and Accuracy $(\mathrm{Acc})=(\mathrm{TP}+\mathrm{TN}) /(\mathrm{TP}+\mathrm{FP}+\mathrm{TN}+\mathrm{FN})$, where TP, TN, FP and FN represent the numbers of true positives, true negatives, false positives and false negatives, respectively. Along with 5-fold cross-validation, different values for the HMMER bit score were also tested in order to obtain the optimal threshold parameter for predicting query sequences. Each value from -20 to 0 was each tested as the HMMER bit score. Then, the results of each fold using each bit score value 
is compared and analyzed. The value which yields the highest cross-validation performance on all five folds of a specific model is selected as the optimal HMMER threshold for that HMM.

\section{Independent testing}

As for classification, the prediction performance of the trained models may be overestimated due to a possible over-fit in the training set; thus, an independent test is done. The experimental plant phosphorylation sites of UniProtKB/Swiss-Prot [11], which were not included in TAIR9 [17], are regarded as the independent test set and are used to estimate the actual prediction performance. Using a window size of 21-mer, there are 332, 105, and 14 phosphorylation sites for $\mathrm{S}$, $\mathrm{T}$, and $\mathrm{Y}$, respectively. Similar to the extraction of a negative set of training data, there are 664, 210, and 28 non-phosphorylation sites for $\mathrm{S}, \mathrm{T}$, and $\mathrm{Y}$, respectively, are regarded as the negative set of independent test data. A balanced number of negative data is selected using K-means clustering to match the number of positive data. After performing a five-fold cross-validation evaluation, the independent test set is used to evaluate the MDD-clustered HMMs with the highest accuracy. After searching against all HMMs, the prediction result for each test data on each HMM is evaluated. If a query data is predicted by at least one HMM to be positive, then it is reported by PlantPhos as a phosphorylation site. This is because each HMM represents the target motif of a specific plant protein kinase which means that a positive hit of a certain model matches the motif it represents. In case where a query data is predicted by two or more HMMs as a phosphorylation site, the cluster which gives the highest prediction score is treated as the plant protein kinase motif that the query data matches. Meanwhile, if a query data is predicted by all HMMs to be negative, then it is considered as a nonphosphorylation site. In order to justify the results due to the balancing of the positive and negative data, the independent testing is done 10 times on $\mathrm{S}, \mathrm{T}$, and $\mathrm{Y}$ data.

\section{Results and discussion}

\section{Investigation of substrate site specificities}

This work aims to investigate the various substrate site specificities in the plant species Arabidopsis thaliana based on amino acid sequences. According to a previous mass spectrometry-based identification of plant phosphorylation sites, a total of 2506, 378, and 108 nonredundant data for $\mathrm{S}, \mathrm{T}$, and $\mathrm{Y}$, respectively, are extracted from the TAIR9 database [17] through its authors. As presented in Table 2, the Two Sample Logo [33] indicates that phosphoserine (pSer), phosphothreonine (pThr), and phosphotyrosine (pTyr) sites contain various conserved amino acids as opposed to non-phosphorylated $\mathrm{S}, \mathrm{T}$, and $\mathrm{Y}$. In order to further investigate different substrate site specificities from the available data, this work applies MDD to cluster all phosphorylated fragments into subgroups that capture the most significant dependencies of amino acid composition between positions. Phosphorylated sequences in each MDD-clustered subgroup which shows a conserved motif, represents particular substrate site specificity. The flanking amino acids $(-10 \sim+10)$ of the non-redundant phosphorylation sites, which are centered on position 0 , are graphically visualized as sequence logos using WebLogo [30,34].

Maximal dependence decomposition is executed multiple times with varying values in order to obtain the most optimal minimum cluster size. Setting the minimum cluster size to 200 for $\mathrm{S}$ data yielded 22 small groups. However, by further analyzing these groups through its corresponding entropy plots, it is observed that several groups contain very similar motifs. These groups are then combined together and visualized using WebLogo. The resulting entropy plots of the combined group exhibit the same motif as the smaller groups. Therefore, the groups were further tuned by combining similar groups into one group. This resulted in 9 subgroups for $\mathrm{S}$. For $\mathrm{T}$ and $\mathrm{Y}$ data, the minimum cluster size was set to 100 , and 30 , respectively, which resulted to 6 subgroups containing distinct motifs for both data sets. The conservation of flanking amino acids in each MDD-clustered subgroup

Table 2 Two Sample Logo in plant phosphoserine, phosphothreonine, and phosphotyrosine

\begin{tabular}{cccc}
\hline Phosphorylated Residue & Number of Non-redundant data & Entropy Plot of Sequence Logo \\
\hline $\mathrm{S}$ & 2506 & & \\
\hline $\mathrm{T}$ & 378 & & \\
\hline $\mathrm{Y}$ & 108 & \\
\hline
\end{tabular}


is represented using entropy plot of sequence logo. Table 3 shows the number of positive data in each subgroup of pSer and their corresponding predictive performances based on a five-fold cross-validation evaluation. According to the chi-square test of the dependence of five amino acid groups in flanking positions (see Additional file 1, Table S1), eight subgroups contain conserved motifs at a specific position. Subgroup S1 reveals that the most pronounced feature of plant pSer sites is the abundance of proline residue at position +1 . Moreover, another significant substrate specificity is observed in subgroup S2 (786 pSer sequences) which contains a statistically conserved motif of negatively charged amino acids ( $D$ and $E$ ) at positions +3 . Five out of all MDD-clustered subgroups contain conserved motifs of positively charged amino acids ( $\mathrm{K}$ and $\mathrm{R})$ at a specific position. In particular, subgroup S5 contains a conserved proline motif at position -9, which is distant to the phosphorylation site (position 0 ). Also, subgroups S3 and S8 contain conserved motifs at distant positions +9 and +10 , respectively. It is observed that subgroup S9 does not contain significantly conserved amino acid at any position.

The MDD-clustered subgroups of pThr and pTyr are presented in Table 4 and 5, respectively. In plant pThr sites, subgroup $\mathrm{T} 1$ also has the abundance of proline residue at position +1 . Another featured motif of $\mathrm{pThr}$ is in subgroup T3 which contains the statistically significant conservation of amino acid residues (D, E, N and Q) at positions +5 . Additionally, subgroups T2, T4, and T5 contain conserved motifs at distant positions $-7,+10$, and +9 , respectively. For plant pTyr sites, subgroup Y3 contains conserved motif of basic amino acids (K, R and $\mathrm{H})$ at position -6; meanwhile, subgroups $\mathrm{Y} 4$ and $\mathrm{Y} 5$ contain conserved motifs of basic residues ( $K$ and $R$ ) and imino residue $(\mathrm{P})$ at positions -1 and +2 , respectively. Both T6 and Y6 do not contain significantly conserved amino acid at any position as observed in the entropy plot motif of Table 4 and Table 5, respectively. Although the application of MDD on 108 pTyr sequences could result in six subgroups, the number of data is deemed insufficient to investigate representative significant motifs. In order to further investigate $S 9$ and T6, MDD clustering is re-applied on these subgroups. As shown in Additional file 1, Table S6, potential substrate specificities can be found within S9 and T6. However, the number of data on each potential substrate motif found is too small to support its validity. This may be improved as additional plant phosphorylation data are acquired.

Table 3 The substrate site specificity and predictive performance in nine MDD-clustered subgroups of phosphoserine

\begin{tabular}{|c|c|c|c|c|c|c|c|}
\hline Group & Number of data & Entropy plot of substrate motif & HMMER bit score & Pre & Sn & Sp & Acc \\
\hline S1 & 624 & i) & -3 & $89.8 \%$ & $90.6 \%$ & $89.7 \%$ & $90.2 \%$ \\
\hline S2 & 786 & & -4 & $76.6 \%$ & $71.9 \%$ & $78.1 \%$ & $75.0 \%$ \\
\hline S3 & 355 & : & -7 & $72.1 \%$ & $68.9 \%$ & $73.2 \%$ & $710 \%$ \\
\hline S4 & 230 & & -6 & $90.3 \%$ & $93.0 \%$ & $90.0 \%$ & $91.5 \%$ \\
\hline S5 & 77 & A & -12 & $76.9 \%$ & $75.0 \%$ & $74.3 \%$ & $74.5 \%$ \\
\hline S6 & 93 & & -10 & $90.2 \%$ & $88.0 \%$ & $90.4 \%$ & $89.1 \%$ \\
\hline S7 & 109 & 1 & -8 & $90.8 \%$ & $83.3 \%$ & $91.7 \%$ & $87.5 \%$ \\
\hline S8 & 61 & 1 & -12 & $77.7 \%$ & $85.0 \%$ & $80.2 \%$ & $82.7 \%$ \\
\hline \multirow[t]{2}{*}{ S9 } & 171 & 1 & -6 & $79.6 \%$ & $83.1 \%$ & $78.5 \%$ & $80.8 \%$ \\
\hline & & 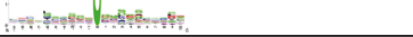 & & & & & \\
\hline Average & & & & $82.6 \%$ & $82.0 \%$ & $82.9 \%$ & $82.4 \%$ \\
\hline
\end{tabular}


Table 4 The substrate site specificity and predictive performance in six MDD-clustered subgroups of phosphothreonine

\begin{tabular}{|c|c|c|c|c|c|c|c|}
\hline Group & Number of data & Entropy plot of substrate motif & HMMER bit score & Pre & Sn & Sp & Acc \\
\hline T1 & 43 & || & -16 & $79.4 \%$ & $74.1 \%$ & $81.1 \%$ & $77.7 \%$ \\
\hline $\mathrm{T} 2$ & 88 & i & -9 & $89.4 \%$ & $91.9 \%$ & $88.6 \%$ & $90.3 \%$ \\
\hline T3 & 77 & $\|$ & -9 & $82.1 \%$ & $80.3 \%$ & $81.9 \%$ & $81.1 \%$ \\
\hline T4 & 34 & $\left.\right|_{\cos 20} \mid$ & -15 & $78.6 \%$ & $76.6 \%$ & $77.1 \%$ & $76.5 \%$ \\
\hline T5 & 42 & 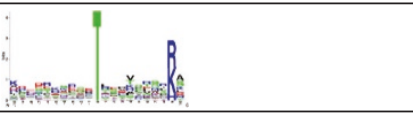 & -13 & $79.4 \%$ & $71.1 \%$ & $81.1 \%$ & $75.9 \%$ \\
\hline T6 & 94 & $\mid$ & -8 & $74.9 \%$ & $61.0 \%$ & $79.8 \%$ & $70.5 \%$ \\
\hline Average & & & & $80.6 \%$ & $75.8 \%$ & $81.6 \%$ & $78.6 \%$ \\
\hline
\end{tabular}

\section{Predictive performance of five-fold cross-validation}

The cross-validation process includes the selection of the threshold parameter for each model. The threshold parameter is a specific bit score that serves as the cutoff value of HMMsearch for determining matching query sequences for an HMM [31]. The threshold is selected by first testing each value from the range of -20 to 0 as the bit score. The threshold is tuned to a specific value which allows an HMM to yield a high and balanced Specificity and Sensitivity for a specific HMM. Table 3 shows the threshold score selected for each model of pSer as well as its individual predictive performance.
Also, Table 4 and 5 show the threshold score selected for each model of pThr and pTyr, respectively, as well as its individual predictive performance.

According to a five-fold cross-validation evaluation, the predictive performance of MDD-clustered HMM performs significantly better than the non-MDD-clustered HMM of pSer, pThr, and pTyr. As shown in Table 6, the single HMM for pSer yields a Precision rate of $49.5 \%$, a Sensitivity rate of $58.6 \%$, a Specificity rate of $70.0 \%$, and an Accuracy rate of $66.2 \%$. On the other hand, the performance of HMM for pSer with MDD clustering yields a Precision rate of $82.6 \%$, a Sensitivity rate of $82.0 \%$, a Specificity rate

Table 5 The substrate site specificity and predictive performance in six MDD-clustered subgroups of phosphotyrosine

\begin{tabular}{|c|c|c|c|c|c|c|c|}
\hline Group & Number of data & Entropy plot of substrate motif & HMMER bit score & Pre & Sn & $\mathrm{Sp}$ & Acc \\
\hline Y1 & 11 & & -16 & $93.3 \%$ & $90.0 \%$ & $90.0 \%$ & $90.0 \%$ \\
\hline Y2 & 7 & & -14 & $80.0 \%$ & $80.0 \%$ & $100 \%$ & $90.0 \%$ \\
\hline Y3 & 15 & & -18 & $85.0 \%$ & $90.0 \%$ & $86.6 \%$ & $85.0 \%$ \\
\hline Y4 & 20 & & -17 & $90.0 \%$ & $90.0 \%$ & $90.0 \%$ & $90.0 \%$ \\
\hline Y5 & 16 & & -16 & $90.0 \%$ & $86.6 \%$ & $88.3 \%$ & $87.1 \%$ \\
\hline Y6 & 39 & & -16 & $93.3 \%$ & $92.1 \%$ & $92.5 \%$ & $92.4 \%$ \\
\hline & & - & & & & & \\
\hline Average & & & & $88.6 \%$ & $88.1 \%$ & $91.2 \%$ & $89.0 \%$ \\
\hline
\end{tabular}


Table 6 The comparison of five-fold cross-validation between single HMM and MDD-clustered HMMs

\begin{tabular}{cccccc}
\hline Method & $\begin{array}{c}\text { Phosphorylated } \\
\text { residue }\end{array}$ & Pre & Sn & Sp & Acc \\
\hline Single HMM & $\mathrm{S}$ & $49.5 \%$ & $58.6 \%$ & $70.0 \%$ & $66.2 \%$ \\
\cline { 2 - 6 } & $\mathrm{T}$ & $45.4 \%$ & $60.5 \%$ & $63.4 \%$ & $62.5 \%$ \\
\hline \multirow{3}{*}{$\begin{array}{c}\text { MDD-clustered } \\
\text { HMMs }\end{array}$} & $\mathrm{Y}$ & $75.4 \%$ & $90.6 \%$ & $84.7 \%$ & $86.6 \%$ \\
\cline { 2 - 6 } & $\mathrm{S}$ & $82.6 \%$ & $82.0 \%$ & $82.9 \%$ & $82.4 \%$ \\
& $\mathrm{~T}$ & $80.6 \%$ & $75.8 \%$ & $81.6 \%$ & $78.6 \%$ \\
\cline { 2 - 6 } & $\mathrm{Y}$ & $88.6 \%$ & $88.1 \%$ & $91.2 \%$ & $89.0 \%$ \\
\hline
\end{tabular}

of $82.9 \%$, and an Accuracy rate of $82.4 \%$. With regard to pThr, using a single HMM yields a Precision rate of $45.4 \%$, a Sensitivity rate of $60.5 \%$, a Specificity rate of $63.4 \%$, and an Accuracy rate of $62.5 \%$. On the other hand, the performance of MDD-clustered HMM for pThr yields a Precision rate of $80.6 \%$, a Sensitivity rate of $75.8 \%$, a Specificity rate of $81.6 \%$, and an Accuracy rate of $78.6 \%$. With regard to $\mathrm{pTyr}$, using a single HMM yields a Precision rate of $75.4 \%$, a Sensitivity rate of $90.6 \%$, a Specificity rate of $84.7 \%$, and an Accuracy rate of $86.6 \%$. On the other hand, the performance of HMM for pTyr with MDD clustering yields a Precision rate of $88.6 \%$, a Sensitivity rate of $88.1 \%$, a Specificity rate of $91.2 \%$, and an Accuracy rate of $89.0 \%$. This results show that HMM for pSer, pThr, and pTyr which utilizes MDD performs significantly better than using a single HMM without MDD clustering.

Evaluation of the selected models using independent test set

Owing to a possible over-fit of the training set used in PlantPhos, the method was further evaluated by using an independent data set. The independent testing data was tested on each HMM model using the selected threshold score. Independent testing is done 10 times on pSer, pThr, and pTyr data in consideration for the balancing of the positive and negative testing data. The independent performance of each pSer model is shown on Additional file 1, Table S2; each pThr model on Additional file 1, Table S3; and each pTyr model on Additional file 1, Table S4. Moreover, as shown in Table 7, the method yields a significantly high sensitivity rate with $81.4 \%$ for MDD-clustered pSer models; $77.1 \%$ for MDD-clustered pThr models; and $83.7 \%$ for MDDclustered pTyr models. On the other hand, it is also observed that the method yields slightly low specificity on the independent test data with a rate of $71.6 \%$ for MDD-clustered pSer models, 69.7\% for MDD-clustered pThr models, and $68.7 \%$ for MDD-clustered pTyr models. The slightly high rate of False Positive predictions could be explained by the number of models we have for pSer, pThr, and pTyr. It is observed that although each model yields a high predictive specificity $(90 \%)$, the existence of a large number of models may induce higher false positive predictions (see Additional file 1, Figure S2). Without the annotation of the corresponding kinase on each phosphorylation site, each query data is predicted based on observed substrate site specificities represented by MDD-clustered HMMs. As discussed above, if a query data is predicted by at least one of MDD-clustered HMMs to be positive, then it is reported by PlantPhos as a potential phosphorylation site. Meanwhile, if a query data is predicted by all HMM models to be negative, then it is considered as a non-phosphorylation site. Thus, a high false positive rate may be yielded by the prediction system.

Table 7 The comprison of independent testing

\begin{tabular}{|c|c|c|c|c|c|}
\hline Method & Phosphorylated residue & Pre (\%) & Sn (\%) & Sp (\%) & Acc (\%) \\
\hline \multirow[t]{3}{*}{ Single HMM } & $S$ & $52.6 \%$ & $50.6 \%$ & $77.2 \%$ & $68.3 \%$ \\
\hline & $\mathrm{T}$ & $50.0 \%$ & $20.9 \%$ & $89.5 \%$ & $66.6 \%$ \\
\hline & Y & $36.3 \%$ & $28.5 \%$ & $75.0 \%$ & $59.5 \%$ \\
\hline \multirow[t]{3}{*}{ MDD-clustered HMMs } & $\mathrm{S}$ & $74.2 \%$ & $81.4 \%$ & $71.6 \%$ & $76.5 \%$ \\
\hline & $\mathrm{T}$ & $71.8 \%$ & $77.1 \%$ & $69.7 \%$ & $73.4 \%$ \\
\hline & Y & $72.8 \%$ & $83.7 \%$ & $68.7 \%$ & $76.2 \%$ \\
\hline \multirow[t]{3}{*}{ PhosPhAt 3.0} & $S$ & $55.5 \%$ & $61.9 \%$ & $50.6 \%$ & $56.2 \%$ \\
\hline & $\mathrm{T}$ & $55.0 \%$ & $57.1 \%$ & $53.3 \%$ & $55.2 \%$ \\
\hline & Y & $4.0 \%$ & $14.2 \%$ & $78.5 \%$ & $46.4 \%$ \\
\hline \multirow[t]{3}{*}{ NetPhos 2.0} & $S$ & $58.6 \%$ & $71.6 \%$ & $49.3 \%$ & $60.5 \%$ \\
\hline & $T$ & $47.5 \%$ & $36.1 \%$ & $59.6 \%$ & $47.8 \%$ \\
\hline & Y & $44.4 \%$ & $28.5 \%$ & $64.2 \%$ & $46.4 \%$ \\
\hline \multirow[t]{3}{*}{ DisPhos 1.3} & $S$ & $66.8 \%$ & $60.6 \%$ & $69.3 \%$ & $65.0 \%$ \\
\hline & $\mathrm{T}$ & $47.5 \%$ & $36.1 \%$ & $61.2 \%$ & $48.6 \%$ \\
\hline & $Y$ & $57.1 \%$ & $47.5 \%$ & $64.2 \%$ & $55.8 \%$ \\
\hline
\end{tabular}


Furthermore, reasonably assuming that each MDD-clustered HMM used in PlantPhos represents the target motif of a plant protein kinase, a false positive prediction may be considered as a novel phosphorylation site. This is due to the fact that the negative data used in this study are not necessarily non-phosphorylation sites although it has not yet been experimentally identified to be phosphorylation sites.

\section{Comparison with other methods}

Currently, very few prediction methods dedicated to identifying phosphorylation sites in plants have been proposed. Among these are PhosPhAt [15] which uses a SVM classifier and the work of Gao et al. [26] which incorporates protein sequence information, protein disordered regions, integrated k-nearest neighbor, and SVM for predicting phosphorylation sites (see Additional file 1, Table S5). However, only PhosPhAt offers a readily-available web tool, PhosPhAt 3.0, for predicting plant phosphorylation sites. For comparison, the independent test data set used for evaluating PlantPhos was utilized. Using a window size of 21-mer, 332 pSer, $105 \mathrm{pThr}$, and 14 pTyr sites were used as the positive test data. A balanced number of negative data is then selected using K-means clustering to match the number of positive data. The resulting data are then classified as phosphorylation or non-phosphorylation sites using both PlantPhos and PhosPhAt 3.0. Finally, the precision, sensitivity, specificity, and accuracy of both methods are calculated in order to compare their respective predictive performance. Table 7 shows the results of both methods after being tested on the same data set. It can be observed that PhosPhAt 3.0 yields an accuracy of $56.2 \%, 55.2 \%$, and $46.4 \%$ for predicting $\mathrm{S}, \mathrm{T}$, and $\mathrm{Y}$ sites, respectively, which is lower as compared to the proposed method which yields an accuracy of $76.5 \%, 73.4 \%$, $76.2 \%$ for predicting $\mathrm{S}, \mathrm{T}$, and $\mathrm{Y}$ sites, respectively.

Moreover, the predictive performance of PlantPhos is compared with general phosphorylation prediction tools in order to compare its ability in identifying plant phosphorylation sites. NetPhos is a general phosphorylation prediction tool trained on mammalian phosphoproteins which uses an artificial neural network method to predict phosphorylation sites in query sequences [35]. DisPhos is another general phosphorylation prediction tool trained on experimentally verified phosphorylation sites which uses disorder information to improve prediction accuracy [36]. Furthermore, DisPhos provides an Arabidopsis thaliana predictor, which is selected for this test. These two phosporylation tools, which are available online, are tested using the same independent data set used for evaluating PlantPhos. Table 7 shows that NetPhos yields an accuracy of $60.5 \%, 47.8 \%$, and $46.4 \%$ for predicting $\mathrm{S}, \mathrm{T}$, and $\mathrm{Y}$ sites. On the other hand, DisPhos
1.3 [36] yields an accuracy of $65.0 \%, 48.6 \%$, and $55.8 \%$ for predicting $\mathrm{S}, \mathrm{T}$, and $\mathrm{Y}$ sites.

\section{Comparison of motifs between MDD-clustered subgroups and well-known kinase groups}

In order to further investigate the significance of each MDD-generated motif that represents its corresponding substrate specificity, each MDD-generated motif is compared with the motifs of known kinase-specific motifs. In order to obtain known kinase-specific motifs, all available kinase-specific phosphorylation sites of multiple organisms were obtained from Phospho.ELM [9] and were integrated together to generate a set of known kinase-specific motifs. The resulting MDD motifs generated in this study were then mapped to the kinase-specific motifs generated from the available data in Phospho.ELM. According to the chisquare test of the dependence of five amino acid groups in flanking positions of plant pSer, the most featured motif is the group that contains conserved proline $(\mathrm{P})$ at position +1 , which is highly similar to two well-known kinase groups in Phospho.ELM, Mitogen Activated Protein Kinase (MAPK) and Cyclin Dependent Kinase (CDK). From the pSer subgroups, it is observed that S1 shows a conserved $\mathrm{P}$ at position +1 ; thus, it can be matched with MAPK and CDK. Furthermore, it is observed that S2 contains a conserved Glutamic Acid (G) and Aspartic Acid (D) at positions $+1,+2$ and +3 . A similar conservation is seen in the Casein kinase II (CK2) family of non-plant organisms in Phospho.ELM. Thus, the pSer group S2 can be matched with CK2. Additionally, the conserved Arginine (A) and Lysine (K) at position -3 in the pSer group $\mathrm{S} 4$ is observed to be similar with the CAMK family 2 (CAMK2) of non-plant organisms in Phospho.ELM; therefore, S4 can be matched with CAMK2. Overall, as presented in Table 8, plant pSer groups $\mathrm{S} 1$ is matched to kinase groups MAPK and CDK, as well as S2 and S4 are matched to kinase groups CK2 and CAMK2, respectively. Similar to the matches in pSer subgroup S1, pThr subgroups T1 is matched to kinase groups MAPK and CDK for having similar conserved amino acids at the same position. Additionally, subgroup T3 is matched to the kinase group CK2.

\section{Implementation of the prediction scheme}

Due to the time-consuming and laboratory-intensive nature of experimental identification, even though a protein can be phosphorylated, precisely identifying the phosphorylation sites on the plant substrate proteins is difficult. Therefore, an effective prediction tool should be developed to identify phosphorylation sites with its substrate site specificity. Following the evaluation by cross-validation and independent testing, the MDD-clustered HMMs are utilized in the construction of webbased prediction system. Users can submit their 
Table 8 The MDD-clustered subgroups matched to the well-known kinase groups in non-plant organisms of Phospho. ELM

\begin{tabular}{|c|c|c|c|c|}
\hline Group & Number of data & Entropy plot of motif & Matched kinase & Entropy plot of kinase motif \\
\hline \multirow[t]{2}{*}{ S1 } & 624 & (1) & MAPK & \\
\hline & & & CDK & \\
\hline S2 & 786 & & CK2 & \\
\hline S4 & 230 & & CAMK2 & \\
\hline \multirow[t]{2}{*}{ T1 } & 43 & in & MAPK & \\
\hline & & & CDK & \\
\hline T3 & 77 & I & CK2 & $6-$ \\
\hline
\end{tabular}

uncharacterized protein sequences in the system; consequently, the web tool, PlantPhos, returns the predicted sites together with the position of the predicted site, the flanking amino acids, the matched MDD-clustered substrate motif, and the prediction score. To demonstrate the performance of the proposed tool, two experimentally verified phosphorylated plant proteins which do not exist in the training data set was studied. The first case study is performed using AT5G05040.1 which is a Cysteine protease inhibitor protein from Arabidopsis thaliana obtained from the Plant Protein Phosphorylation Database [16]. As shown in Figure 2(A), AT5G05040.1 contains 2 experimentally verified pSer at positions 36 and 43, and an experimentally verified pTyr at position 34. PlantPhos is able to correctly predict the experimentally verified pSer at positions 36 and 43 as well as the experimentally verified pTyr at position 34 . Moreover, three more $S$ residues are reported by PlantPhos as novel phosphorylated sites. Next, in order to evaluate the performance of PlantPhos in identifying phosphorylation sites from a different plant species aside from Arabidopsis thaliana, the second case study is performed using Medtr6g031130.1 which is a Remorin, N-terminal region protein from Medicago Truncatula obtained from the Plant Protein Phosphorylation Database [16]. As shown in Figure 2(B), Medtr6g031130.1 contains 1 experimentally verified pSer at positions 76. PlantPhos is able to correctly predict the experimentally verified pSer at position 76 .
Additionally, one more $\mathrm{S}$ and two $\mathrm{T}$ are reported by PlantPhos as novel phosphorylated sites.

\section{Conclusions}

The importance of phosphorylation has been indicated in the regulation of protein functions and cell signaling, but the state of research in this field is hindered by experimental difficulties especially for the investigation of substrate site specificity in various organisms. In this work, we have analyzed plant phosphorylation sites by applying MDD clustering; using this method, the available plant phosphorylation data were grouped into several subgroups-each showing a significant conserved motif. Then, we developed a novel method for predicting protein phosphylation sites in plants by training HMMs for each MDD-clustered subgroup which are then used to predict potential phosphorylation sites by HMMsearch. Our method is evaluated by means of 5fold cross-validation which yields an average accuracy of $82.4 \%$ for predicting pSer, $78.6 \%$ for predicting pThr, and $89.0 \%$ for predicting pTyr. Moreover, our method is further evaluated by testing it on an independent data set which shows that our method can predict novel phosphorylation sites by using the experimental phosphorylation data in plant proteins from UniProtKB/ SwissProt. Additionally, we were able to further investigate the MDD-clustered motifs in plants by referencing to the motifs of known kinases from Phospho.ELM [9]. Through this method, we were able to observe similar 


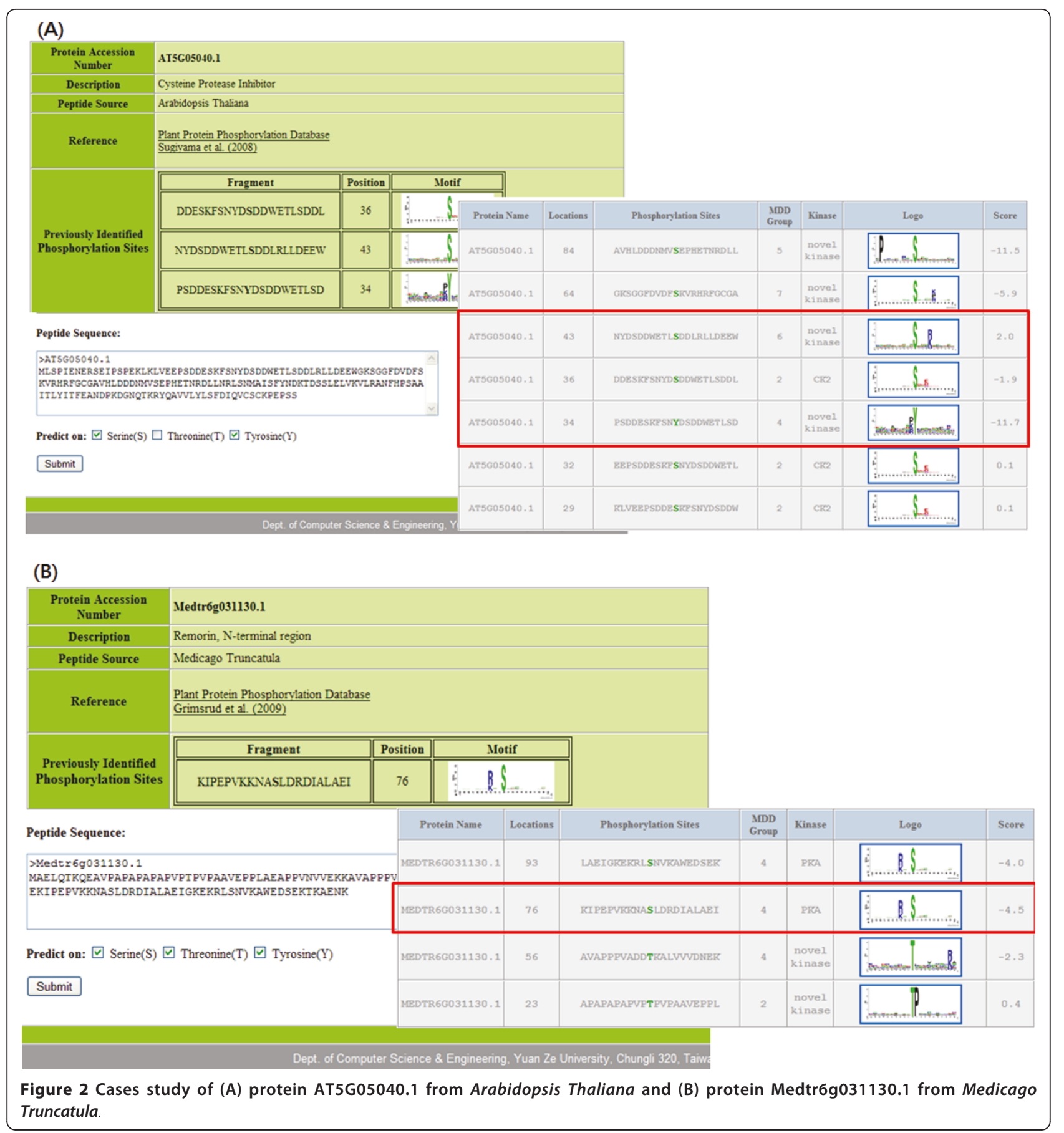

kinase motifs between plants and other organisms. Lastly, the method has been implemented as a web tool named PlantPhos in order for the research community to be able to facilitate phosphorylation site prediction on plant protein data using the proposed method.

Future development for PlantPhos involves (i) the extension of the system to include other plant species other than Arabidopsis thaliana; (ii) the acquisition of additional experimentally verified plant protein data to re-calibrate more robust MDD clusters; (iii) and a more comprehensive investigation of substrate site specificities in plants with additional plant phosphorylation data.

\section{Availability}

PlantPhos can be accessed via a web interface, and is freely available to all interested users at http://csb.cse. yzu.edu.tw/PlantPhos/. All of the data set used in this work is also available for download in the website. 


\section{Additional material}

Additional file 1: Additional Figures and Tables. Contains additional Figures and Tables showing further results in the study

\section{Acknowledgements}

The authors sincerely appreciate the National Science Council of the Republic of China for financially supporting this research under Contract Numbers of NSC 99-2320-B-155-001.

\section{Authors' contributions}

TYL conceived and supervised the project. NAB was responsible for the design, computational analyses, implemented the web-based tool, and drafted the manuscript with revisions provided by TYL and CTL. All authors read and approved the final manuscript.

\section{Competing interests}

The authors declare that they have no competing interests.

Received: 14 March 2011 Accepted: 26 June 2011

Published: 26 June 2011

\section{References}

1. Steen $\mathrm{H}$, Jebanathirajah JA, Rush J, Morrice N, Kirschner MW: Phosphorylation analysis by mass spectrometry: myths, facts, and the consequences for qualitative and quantitative measurements. Mol Cell Proteomics 2006, 5(1):172-181.

2. Hubbard MJ, Cohen P: On target with a new mechanism for the regulation of protein phosphorylation. Trends Biochem Sci 1993, 18(5):172-177.

3. Steffen M, Petti A, Aach J, D'Haeseleer P, Church G: Automated modelling of signal transduction networks. BMC Bioinformatics 2002, 3:34

4. Stone JM, Walker JC: Plant protein kinase families and signal transduction. Plant Physiol 1995, 108(2):451-457.

5. Diolez P, Kesseler A, Haraux F, Valerio M, Brinkmann K, Brand MD: Regulation of oxidative phosphorylation in plant mitochondria. Biochem Soc Trans 1993, 21(Pt 3(3)):769-773.

6. Huber SC: Exploring the role of protein phosphorylation in plants: from signalling to metabolism. Biochemical Society Transactions 2007, 35.

7. Luan S: Tyrosine phosphorylation in plant cell signaling. Proc Natl Acad Sci USA 2002, 99(18):11567-11569.

8. Aebersold R, Mann M: Mass spectrometry-based proteomics. Nature 2003, 422(6928): 198-207.

9. Diella F, Gould CM, Chica C, Via A, Gibson TJ: Phospho.ELM: a database of phosphorylation sites-update 2008. Nucleic Acids Res 2008, 36 Database: D240-244.

10. Hornbeck PV, Chabra I, Kornhauser JM, Skrzypek E, Zhang B: PhosphoSite: A bioinformatics resource dedicated to physiological protein phosphorylation. Proteomics 2004, 4(6):1551-1561.

11. Schneider M, Lane L, Boutet E, Lieberherr D, Tognolli M, Bougueleret L, Bairoch A: The UniProtKB/Swiss-Prot knowledgebase and its Plant Proteome Annotation Program. J Proteomics 2009, 72(3):567-573.

12. Gnad F, Ren S, Cox J, Olsen JV, Macek B, Oroshi M, Mann M: PHOSIDA (phosphorylation site database): management, structural and evolutionary investigation, and prediction of phosphosites. Genome Biol 2007, 8(11):R250

13. Lee TY, Hsu JB, Chang WC, Wang TY, Hsu PC, Huang HD: A comprehensive resource for integrating and displaying protein post-translational modifications. BMC Res Notes 2009, 2:111.

14. Lee TY, Huang HD, Hung JH, Huang HY, Yang YS, Wang TH: dbPTM: an information repository of protein post-translational modification. Nucleic Acids Res 2006, 34 Database:D622-627.

15. Heazlewood JL, Durek P, Hummel J, Selbig J, Weckwerth W, Walther D, Schulze WX: PhosPhAt: a database of phosphorylation sites in Arabidopsis thaliana and a plant-specific phosphorylation site predictor. Nucleic Acids Res 2008, 36 Database:D1015-1021.

16. Gao J, Agrawal GK, Thelen JJ, Xu D: P3DB: a plant protein phosphorylation database. Nucleic Acids Res 2009, 37 Database:D960-962.
17. Huala E, Dickerman AW, Garcia-Hernandez M, Weems D, Reiser L, LaFond F, Hanley D, Kiphart D, Zhuang M, Huang W, et al: The Arabidopsis Information Resource (TAIR): a comprehensive database and web-based information retrieval, analysis, and visualization system for a model plant. Nucleic Acids Res 2001, 29(1):102-105.

18. Huang HD, Lee TY, Tzeng SW, Horng JT: KinasePhos: a web tool for identifying protein kinase-specific phosphorylation sites. Nucleic Acids Res 2005, 33 Web Server:W226-229.

19. Huang HD, Lee TY, Tzeng SW, Wu LC, Horng JT, Tsou AP, Huang KT: Incorporating hidden Markov models for identifying protein kinasespecific phosphorylation sites. J Comput Chem 2005, 26(10):1032-1041.

20. Wong YH, Lee TY, Liang HK, Huang CM, Wang TY, Yang YH, Chu CH, Huang HD, Ko MT, Hwang JK: KinasePhos 2.0: a web server for identifying protein kinase-specific phosphorylation sites based on sequences and coupling patterns. Nucleic Acids Res 2007, 35 Web Server:W588-594.

21. Blom N, Sicheritz-Ponten T, Gupta R, Gammeltoft S, Brunak S: Prediction of post-translational glycosylation and phosphorylation of proteins from the amino acid sequence. Proteomics 2004, 4(6):1633-1649.

22. Obenauer JC, Cantley LC, Yaffe MB: Scansite 2.0: Proteome-wide prediction of cell signaling interactions using short sequence motifs. Nucleic Acids Res 2003, 31(13):3635-3641

23. Xue Y, Zhou F, Zhu M, Ahmed K, Chen G, Yao X: GPS: a comprehensive www server for phosphorylation sites prediction. Nucleic Acids Res 2005, 33 Web Server:W184-187.

24. Zhou FF, Xue Y, Chen GL, Yao X: GPS: a novel group-based phosphorylation predicting and scoring method. Biochem Biophys Res Commun 2004, 325(4):1443-1448.

25. Xue Y, Li A, Wang L, Feng H, Yao X: PPSP: prediction of PK-specific phosphorylation site with Bayesian decision theory. BMC Bioinformatics 2006, 7:163.

26. Gao J, Agrawal GK, Thelen JJ, Obradovic Z, Dunker AK, Xu D: A New Machine Learning Approach for Protein Phosphorylation Site Prediction in Plants. Lect Notes Comput Sci 2009, 5462/2009:18-29.

27. Lee TY, Lin ZQ, Hsieh SJ, Bretana NA, Lu CT: Exploiting maximal dependence decomposition to identify conserved motifs from a group of aligned signal sequences. Bioinformatics 2011, 27(13):1780-7, 1.

28. Burge C, Karlin S: Prediction of complete gene structures in human genomic DNA. J Mol Biol 1997, 268(1):78-94

29. Shien DM, Lee TY, Chang WC, Hsu JB, Horng JT, Hsu PC, Wang TY, Huang HD: Incorporating structural characteristics for identification of protein methylation sites. J Comput Chem 2009, 30(9):1532-1543.

30. Crooks GE, Hon G, Chandonia JM, Brenner SE: WebLogo: a sequence logo generator. Genome Res 2004, 14(6):1188-1190.

31. Eddy SR: Profile hidden Markov models. Bioinformatics 1998, 14(9):755-763.

32. Chou KC, Shen HB: Recent progress in protein subcellular location prediction. Anal Biochem 2007, 370(1):1-16.

33. Vacic $V$, lakoucheva LM, Radivojac P: Two Sample Logo: a graphical representation of the differences between two sets of sequence alignments. Bioinformatics 2006, 22(12):1536-1537.

34. Schneider TD, Stephens RM: Sequence logos: a new way to display consensus sequences. Nucleic Acids Res 1990, 18(20):6097-6100.

35. Blom N, Gammeltoft S, Brunak S: Sequence and structure-based prediction of eukaryotic protein phosphorylation sites. J Mol Biol 1999, 294(5):1351-1362.

36. lakoucheva LM, Radivojac P, Brown CJ, O'Connor TR, Sikes JG, Obradovic Z, Dunker AK: The importance of intrinsic disorder for protein phosphorylation. Nucleic Acids Res 2004, 32(3):1037-1049.

doi:10.1186/1471-2105-12-261

Cite this article as: Lee et al:: PlantPhos: using maximal dependence decomposition to identify plant phosphorylation sites with substrate site specificity. BMC Bioinformatics 2011 12:261. 